\title{
Reliability and Concurrent Validity of the International Classification of Diseases 11th Revision (ICD-11) Guidelines for Depressive Severity
}

M. I. Montoya1, C. S. Kogan ${ }^{1,2}$, S. Paterniti ${ }^{2,3,4}$, J. Shlik $2,3,4$, I. Sterner ${ }^{2,4}$

1 University of Ottawa, School of Psychology, Ottawa, Canada.

${ }^{2}$ Royal's Institute of Mental Health Research, affiliated with the University of Ottawa, Ottawa, Canada.

${ }^{3}$ University of Ottawa, Department of Psychiatry; ${ }^{4}$ Royal Ottawa Mental Health Centre, the Royal, Ottawa, Canada

Results

Current treatment guidelines for depression rely on accurate assessment of severity, which informs clinical decisions from watchful waiting, to offering psychotherapy, to initiating antidepressant medications ${ }^{1}$. However, a universal operational definition of depressive severity is lacking ${ }^{2}$.

The World Health Organization (WHO) approved the 11th revision of the International Classification of Diseases and Related Health Conditions (ICD-11) in May 2019. A major goal was improvement of clinical utility of the classification of mental and behavioural disorders.

ICD-11 clinical descriptions and diagnostic guidelines define depressive episode severity as mild, moderate, or severe based on number and severity of symptoms, and functional impact of mood disturbances.

\section{Study Aims}

1. To determine the inter-rater reliability of the ICD-11 severity qualifier for Depressive episode in the context of any mood disorder (e.g., Recurrent Depressive Disorder)

2. To examine concurrent validity between ICD-11 severity ratings and the patient self-report measure, Beck Depression Inventory-2 (BDI-2), and the Hamilton depression rating scale (HAM-D), a clinician administered measure.

\section{Method}

The Royal Ottawa Mental Health Centre is the Canadian site for the WHO's ICD-11 clinic-based field trials for mental and behavioural disorders.

As part of an international effort ${ }^{3}$, the trial was designed to evaluate the inter-rater reliability and clinical utility of the proposed diagnostic guidelines for Mood Disorders, Anxiety and Fear-Related Disorders, and Disorders Specifically Associated with Stress (Figure 1).

\section{Analysis}

Inter-rater reliability between Primary Interviewer and Observer was evaluated using the Kappa statistic.

Chi-square tests were used to evaluate the association between ICD-11 severity ratings (partial/full remission, mild, moderate, and severe) and the four severity levels (minimal, mild, moderate, and severe) of the BDI-2 and the HAM-D, respectively.

\section{Figure 1. Canadian field trial procedures}

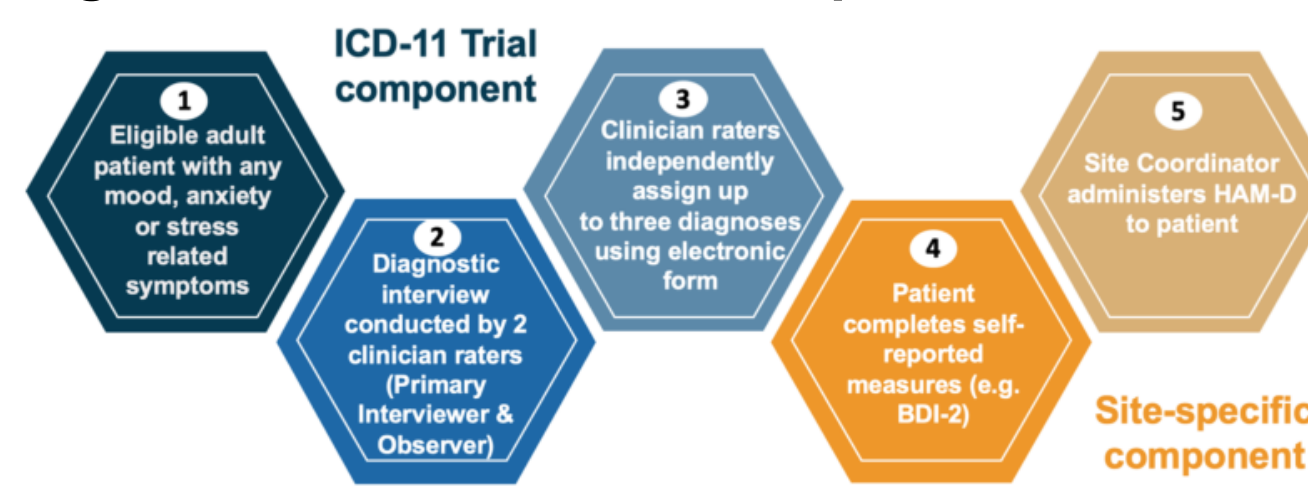

Kennedy SH, Lam RW, McIntyre RS, Tourjman SV, Bhat V, Blier P, et al Canadian Network (a) Management of Adults with Major Depressive Disorder: Section 3. Pharma
Treatments. The Canadian Journal of Psychiatry. 2016 Sep;61(9):540-60

Zimmerman M, Morgan TA, Stanton K. The severity of psychiatric disorders: World Psychiatry. World Psychiatry. 2018 Oct;17(3):258-75.
- A total of 7 clinicians (2 Psychiatrists, 5 Psychologists) served as clinician raters.

- A total of 102 patients were assessed for the field trial, of which 70 (mean age: $41.5 \pm 14.2$; female: 49 ) received a Depressive episode diagnosis from both clinician raters. Primary Interviewer and Observer assigned a total of 77 Depressive episode diagnoses independently.

Inter-rater Reliability

Table 1. Reliability of depressive episode severity qualifier $(\mathrm{N}=70)$

\begin{tabular}{|l|c|c|c|}
$\begin{array}{c}\text { Depressive episode } \\
\text { diagnosis }\end{array}$ & $\begin{array}{c}\text { Joint rater } \\
\text { agreement } \\
\text { (Intraclass K) }\end{array}$ & $\begin{array}{c}\text { Standard } \\
\text { error }\end{array}$ & $95 \% \mathrm{Cl}$ \\
\hline Partial/Full-remission & .824 & .068 & $.690-.940$ \\
\hline Mild & $-.061^{*}$ & .026 & $-.109-.000$ \\
\hline Moderate & .229 & .118 & $.003-.458$ \\
\hline Severe & .353 & .150 & $.043-.638$
\end{tabular}

$*=p>.05$

Kappa (k) interpretation ${ }^{4}$ : slight 0-0.2, fair 0.21-0.4, moderate $0.41-0.60$, substantial 0.61-0.80, almost perfect 0.81-1.0.

Table 2. Breakdown of Primary Interviewer and Observer agreement on depressive episode severity qualifier

\begin{tabular}{l|l|c|c|c|c|c|}
\multicolumn{2}{c|}{} & \multicolumn{6}{|c}{ Observer } \\
\cline { 3 - 8 } & $\begin{array}{c}\text { Partial/Full- } \\
\text { remission }\end{array}$ & Mild & Moderate & Severe & Total \\
\hline \multirow{4}{*}{$\begin{array}{l}\text { Primary } \\
\text { Interviewer }\end{array}$} & $\begin{array}{l}\text { Partial/Full } \\
\text {-remission }\end{array}$ & 26 & 0 & 1 & 0 & 27 \\
\cline { 2 - 8 } & Mild & 1 & 0 & 5 & 0 & 6 \\
\cline { 2 - 8 } & Moderate & 4 & 3 & 13 & 6 & 26 \\
\cline { 2 - 8 } & Severe & 0 & 0 & 6 & 5 & 11 \\
\cline { 2 - 8 } & Total & 31 & 3 & 25 & 11 & 70
\end{tabular}

= exact agreement

- Weighted Kappa $\left(\mathrm{K}_{\mathrm{w}}\right)$ of overall severity levels indicated significant agreement $\mathrm{K}_{\mathrm{w}}=.646,95 \% \mathrm{Cl}[.525, .767], \mathrm{p}<.001$

\section{Concurrent Validity}

There was a statistically significant association between clinician-rated severity and:

- $\quad$ BDI-2 severity $X^{2}(9)=28.457, p=.001$, with a medium effect size, Cramer's $\mathrm{V}=.351$

- HAM-D severity $X^{2}(9)=27.362, p=.002$, with a medium effect size, Cramer's V $=.344$.

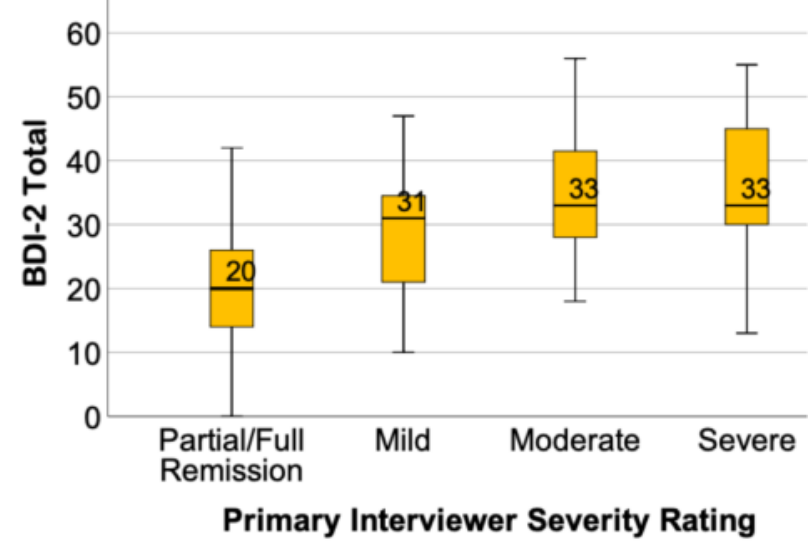

Figure 2. BDI-2 total score by ICD11 depressive severity

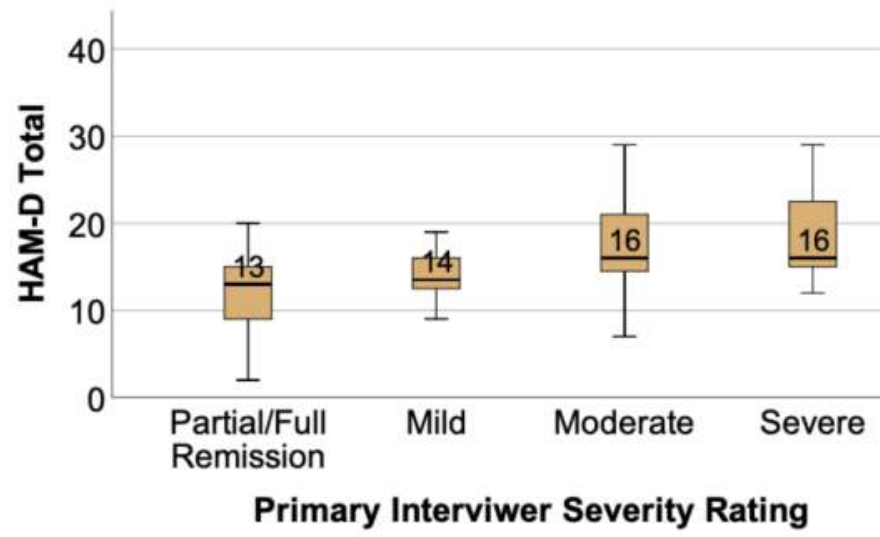

Figure 3. HAM-D total score by ICD11 depressive severity

\section{Discussion}

Reliability of partial/full remission was considered almost perfect. Severity qualifiers were considered less than fair, with mild being the least reliable, likely due to low $\mathrm{N}$ for this level. When considering the degree of disagreement, reliability across all four ratings was considered substantial. Other aspects of each case may have affected determination of severity by clinicians.

Concurrent validity results support the conceptualization of Depressive episode severity proposed in ICD-11. 